\title{
Optimización de la Eficiencia Térmica de un Motor Robinson Aplicando el Modelo Senft-Schmidt-Petrescu
}

\author{
Ignacio Carvajal $^{(1)}$, Georgiy Polupan ${ }^{(1)}$, Guillermo Jarquin ${ }^{(2)}$, Jorge Vázquez $^{(3)}$ \\ (1) Instituto Politécnico Nacional, Esc. Superior de Ing. Mecánica y Eléctrica, Unid. Prof. Zacatenco. Av. IPN \\ s/n, Edif. 5, 07738 México D.F., México (e-mail: icarvajal@ipn.mx, gpolupan@ipn.mx) \\ (2) Instituto Politécnico Nacional, Esc. Superior de Ing. Mecánica y Eléctrica, Unid.Prof.Culhuacan. Av. \\ Santa Ana No. 1000, Edif.2, 3er piso, 04430 México, D.F., México(e-mail: gjarquin@ipn.mx) \\ (3) UNAM, Lab. de Térmica y Fluidos, Fac. de Estudios Superiores, Aragón. Av. Rancho Seco s/n, Col. \\ Impulsora, C.P. 57130, Estado de México, MÉXICO(e-mail: antoni34@todito.com.mx)
}

Recibido Nov. 26, 2012; Aceptado Ene. 02, 2013; Versión final recibida Mar. 02, 2013

\begin{abstract}
Resumen
Se presentan los resultados de la optimización de la eficiencia térmica de un motor Robinson aplicando el modelo Senft-Schmidt-Petrescu. Este se fundamenta en el acoplamiento de los modelos de Senft-Schmidt y de Petrescu, usados para el cálculo de la potencia en motores de ciclo Stirling. El modelo propuesto involucra tanto parámetros termodinámicos como geométricos y permite estimar en su conjunto las pérdidas de presión por fricción y por estrangulamiento del flujo. Tomando como base los datos del prototipo de un motor Robinson de aproximadamente $2 \mathrm{~W}$ de potencia, se analizaron teóricamente sus parámetros principales y se optimizó su eficiencia térmica en función de la mínima generación de entropía. Después de realizada la optimización, la eficiencia térmica del motor aumentó a más del doble. Se concluye que el modelo Senft-Schmidt-Petrescu propuesto puede ser útil en el análisis y rediseño de motores Robinson.
\end{abstract}

Palabras clave: optimización, eficiencia térmica, modelo matemático, motor Robinson

\section{Optimization of the Thermal Efficiency of a Robinson Engine Applying the Senft-Schmidt-Petrescu Model}

\begin{abstract}
The results of the optimization of the thermal efficiency of a Robinson engine applying the Senft-PetrescuSchmidt model are presented. This is based on the coupling of the Senft-Schmidt and the Petrescu models, used for the calculation of power in Stirling cycle engines. The proposed model involves both thermodynamic and geometric parameters and allows estimating the overall pressure losses by friction and by flow throttling. Based on data from a prototype of a Robinson engine of about $2 \mathrm{~W}$ of power, a theoretical analysis of its main parameters and the optimization of thermal efficiency, based on the minimum entropy generation, were performed. After the optimization was done, the thermal efficiency of the engine increased to more than the double. It is concluded that the proposed Senft-Schmidt-Petrescu model may be useful in the analysis and redesign of Robinson engines.
\end{abstract}




\section{INTRODUCCIÓN}

Los motores térmicos son dispositivos empleados principalmente para la obtención de trabajo y potencia. Los más utilizados actualmente son los motores endotérmicos en sus dos variantes: los motores alternativos y las turbinas de gas. Sin embargo, el constante incremento de los costos de los combustibles fósiles, de los cuales son dependientes estos motores, y el continuo deterioro del medio ambiente, han provocado que se considere nuevamente a los motores exotérmicos, como los motores de ciclo Stirling, según se puede apreciar en la literatura (Ferreira et al., 2012; Karabulut et al., 2009; Khan, 2012; Miranda et al., 2012a; Petrescu et al., 2010; Senft, 2011). Éstos motores presentan un panorama amplio a sus aplicaciones debido a que pueden utilizar fuentes alternas de energía, como la solar, y energías residuales de procesos terminados, como vapor o gases de combustión (Kongtragool y Wongwises, 2008; Santos et al., 2012; Siva Reddy et al., 2012).

Durante el desarrollo inicial de los motores Stirling, las dimensiones de las cámaras del desplazador y del pistón se determinaban de forma empírica pues no había modelos matemáticos. Uno de los primeros esfuerzos en ese sentido, fue el desarrollo del modelo matemático de Schmidt, también llamado teoría de Schmidt, donde se presentan los métodos de cálculo para cada variante de los motores Stirling.

En el modelo de Gustav Schmidt (Chen y Griffin, 1983) se considera que los volúmenes de los espacios de compresión y expansión varían sinusoidalmente en el tiempo. La teoría de Schmidt provee resultados analíticos simples y se toma como el modelo clásico para la operación del motor Stirling ideal. Sin embargo, de acuerdo a De Boer (2003) el modelo de Schmidt no toma en cuenta las pérdidas, por lo que la eficiencia térmica teórica iguala a la eficiencia de Carnot. Debido a esto, los resultados de trabajo, potencia y rendimiento que se obtienen con el modelo de Schmidt están sobreestimados.

Para obtener un modelo matemático que incluyera todas las pérdidas en el motor, el modelo de Schmidt fue ajustado por Finkelstein (Chen y Griffin, 1983), quien consideró por separado a las pérdidas que se presentan en cada parte del motor por lo que recibe el nombre de Método de Análisis Nodal. Sin embargo, existe la dificultad de tener que conocer de antemano cada parte del motor bajo estudio y cada lugar en que se presentará una caída de presión por estrangulamiento de flujo. Por lo general no se cuenta con esta información así que se debe especular sobre los valores de algunos datos lo que conduce a errores en el cálculo. Sin embargo, se ha considerado que el modelo de Schmidt ajustado por Finkelstein es adecuado por tomar en cuenta las irreversibilidades debidas a las caídas de presión por estrangulamiento. Posteriormente se le hizo una mejora al incluir otras caídas de presión, las pérdidas de fluido debidas a fugas y las pérdidas de calor a través de las paredes de las secciones del motor. Aunque el Método de Análisis Nodal se complementó, existen variables que no pueden ser comprobadas de manera experimental y tienen un alto grado de complejidad y de precisión matemática de acuerdo con Organ (1992).

En las últimas décadas, el continuo desarrollo de métodos para el diseño de motores Stirling condujo al modelo de Senft-Schmidt, donde Senft (2002) realizó ajustes al modelo de Schmidt para calcular el valor del trabajo indicado y su representación gráfica, incluyendo los espacios muertos.

Por otra parte, Petrescu retomó la ecuación de la primera ley de la termodinámica con la idea de obtener la relación entre el desarrollo de trabajo y las principales pérdidas de presión que están presentes en los motores Stirling actuales en sus configuraciones $\beta$ y $\gamma$. Con ello aplicó el Método Directo, que usa la integración directa de la ecuación de la primera ley con la presión media indicada del ciclo, considerando irreversibilidades del ciclo con velocidad finita (Petrescu et al., 2002). Las pérdidas de presión que considera el método son las debidas a la fricción mecánica y las debidas al paso del flujo a través del regenerador, mejor conocidas como pérdidas por estrangulamiento. Estas pérdidas se pueden estimar usando la ecuación del trabajo irreversible para procesos con velocidad finita en sistemas cerrados propuesta por el autor mencionado Petrescu et al. (2002). Aunque en este método se desprecia usar el volumen muerto de los motores, hay que resaltar que sí se calcula el trabajo efectuado durante el ciclo termodinámico real o irreversible, $W_{\text {irrev }}$, pero sin conocer el valor de las pérdidas de forma directa.

Recientemente, Cheng y Yang (2012) usaron el modelo reversible para obtener la combinación óptima entre el ángulo de fase y la relación del volumen barrido para maximizar el trabajo del eje de los motores Stirling en sus tres configuraciones principales $\alpha, \beta$ y $\gamma$. El modelo reversible no considera las irreversibilidades, por ejemplo, las pérdidas de presión. Por lo tanto, no se puede usar para evaluar las irreversibilidades directa o indirectamente. Por otra parte, Campos et al. (2012) realizaron la optimización termodinámica del ciclo de un motor de cuatro cilindros de doble actuación, aplicando un modelo matemático dinámico adimensional. En el modelo empleado se desprecian las pérdidas de presión en el regenerador, es decir, las pérdidas por estrangulamiento, lo que limita la aplicación de este modelo sólo a cierto de tipo de motores de ciclo Stirling donde el regenerador provoca una muy baja pérdida de presión o no tienen regenerador. 
Otras investigaciones recientes están específicamente relacionadas con el modelado y estudio experimental de los motores Stirling tipo $\alpha$ (Khan, 2012; Siva Reddy et al., 2012) y $\beta$ (Karabulut et al., 2009; Kongtragool y Wongwises, 2008). Sin embargo, son pocas las publicaciones que se refieren específicamente al estudio teórico-experimental de los motores Stirling tipo $\gamma$ y del motor Robinson (Gheith et al., 2012; Miranda et al., 2012b). Estas últimas dos configuraciones como se sabe tienen la ventaja de operar entre la menor diferencia de temperaturas (Cheng y Yang, 2012; Ferreira et al., 2012; Gheith et al., 2012) lo que los hace atractivos para su aplicación en la recuperación de calor residual de los procesos térmicos.

Por lo anteriormente expuesto, la motivación de este trabajo es desarrollar un modelo matemático relativamente sencillo que involucre tanto parámetros termodinámicos como geométricos, además de estimar en su conjunto las pérdidas de presión por fricción y por estrangulamiento del flujo. Este modelo estará dirigido al análisis teórico de los parámetros principales de un motor Robinson con el fin de optimizar su eficiencia térmica en función de la mínima generación de entropía.

\section{DESARROLLO DEL MODELO MATEMÁTICO DE SENFT-SCHMIDT-PETRESCU}

Al comparar los modelos matemáticos descritos en la introducción, se puede notar que al aplicar el Método Directo [Petrescu et al., 2002] se obtiene una ecuación del trabajo útil del ciclo, el cual se considera para sistemas cerrados como equivalente al trabajo indicado o irreversible. Esto es válido ya que se toman en cuenta las pérdidas de presión involucradas y el trabajo reversible pero no al volumen muerto total en el motor. Por el contrario, el modelo matemático de Senft (2002) presenta como resultado una ecuación para el cálculo del trabajo indicado considerando el volumen muerto pero no las pérdidas. De esta forma, es posible crear una ecuación que involucre ambas aproximaciones:

$$
\mathrm{W}_{\mathrm{ind}}=\mathrm{W}_{\mathrm{rev}}+\mathrm{W}_{\mathrm{perd}}
$$

La parte izquierda de la igualdad es el trabajo indicado del ciclo $W_{\text {ind }} y$ la parte derecha es el trabajo del ciclo o trabajo reversible $W_{\text {rev }}$ más las pérdidas generadas $W_{\text {perd }}$. Con esta ecuación, se puede hacer una estimación de las pérdidas involucradas en el motor cuando se obtiene trabajo real, considerando de esta manera, tanto a la geometría del motor Stirling como a los efectos por caídas de presión.

Por lo anteriormente descrito, se propone unir el modelo de Senft (2002) o modelo de Senft-Schmidt y el método directo o modelo de Petrescu et al. (2002) por medio de la ecuación (1), obteniéndose la base de un nuevo modelo matemático que ajusta la geometría de los motores Stirling con su comportamiento térmico. El acoplamiento de las ecuaciones se realiza considerando la primera y segunda leyes de la termodinámica, ya que cuando un sistema opera irreversiblemente se destruye trabajo a un valor que es proporcional a la razón de generación de entropía del sistema [Bejan, 2006]. El trabajo que no se aprovecha debido a las irreversibilidades termodinámicas, $\left(\mathrm{W}_{\text {rev }}-\mathrm{W}_{\text {irrev }}\right)$, es llamado también trabajo disponible no aprovechado.

La proporcionalidad entre trabajo disponible no aprovechado y la generación de entropía, conduce a la ecuación (2) conocida como teorema de Gouy-Stodola o de la mínima generación de entropía [Bejan, 2006]:

$\dot{\mathrm{W}}_{\text {perd }}=\mathrm{T}_{0} \mathrm{~S}_{\text {gen }}$

El modelo matemático propuesto se obtiene al aplicar el teorema de Gouy-Stodola para acoplar la ecuación del trabajo indicado del modelo de Senft (2002) y del trabajo irreversible del modelo de Petrescu et al. (2002). Sustituyendo la ecuación (2) en la ecuación (1), y después de la simplificación se tiene:

$$
\begin{aligned}
& \frac{V_{T} \bar{p}}{\kappa+1} \frac{\pi(1-\tau) \kappa s e n \alpha}{\left(\sqrt{Y^{2}-X^{2}}+Y\right)}=W_{\text {rev }} \pm\left[a\left(\frac{w}{c}\right) \bar{p}_{i} \pm b\left(\frac{\Delta p_{\text {est }}}{2}\right) \pm f\left(\Delta p_{f}\right)\right]\left(V_{2}-V_{1}\right) \\
& \frac{V_{T} \bar{p}}{\kappa+1} \frac{\pi(1-\tau) \kappa s e n \alpha}{\left(\sqrt{Y^{2}-X^{2}}+Y\right)}=W_{\text {rev }}-\left[T_{0} \cdot S_{\text {gen }}\right]
\end{aligned}
$$


$-T_{0} \cdot S_{\text {gen }}=\frac{V_{T} \bar{p}}{\kappa+1} \frac{\pi(1-\tau) \kappa s e n x}{\left(\sqrt{Y^{2}-X^{2}}+Y\right)}-W_{r e v}$

$S_{\text {gen }}=\frac{W_{\text {rev }}-\frac{V_{T} \bar{p}}{\kappa+1} \frac{\pi(1-\tau) \kappa s e n x}{\left(\sqrt{Y^{2}-X^{2}}+Y\right)}}{T_{0}}$

$\mathrm{S}_{\text {gen }}=\frac{\mathrm{W}_{\mathrm{rev}}-\mathrm{W}_{\text {ind }}}{\mathrm{T}_{0}}$

En la ecuación (4) el segundo término en el lado derecho corresponde al trabajo disponible total no aprovechado durante el ciclo térmico. En la ecuación (7), qué se le ha llamado el modelo de Senft-SchmidtPetrescu, se puede notar que si aumenta la temperatura de referencia, $T_{0}$, la entropía generada, $S_{\text {gen }}$, disminuirá siempre y cuando se mantenga constante la relación $\mathrm{W}_{\text {rev }}-\mathrm{W}_{\text {ind. }}$. Precisamente, se propone que esta ecuación sea la comprobación de los valores calculados de la entropía.

\section{MOTOR ROBINSON}

Debido a que se cuenta con las dimensiones y características principales de un motor Robinson de baja potencia (ver Tabla 1) y el hecho de que la información acerca de la optimización de este tipo de motores es escasa, este tipo de motor fue elegido para aplicar el modelo Senft-Schmidt-Petrescu. Sin embargo, la coincidencia en las ecuaciones de cálculo, la configuración del motor Robinson es de cilindros separados, hace que también sea posible la aplicación del modelo Senft-Schmidt-Petrescu en el análisis de motores Stirling tipo $\gamma$.

El motor Robinson consta de dos cilindros separados, en uno de los cuales se sitúa el desplazador y en otro el pistón de potencia, cuyos ejes se encuentran ubicados en un ángulo de aproximadamente $90^{\circ}$. El calentamiento y enfriamiento del fluido de trabajo ocurre en los límites de la cámara del desplazador. El fluido de trabajo es aire atmosférico al cual se le considera como gas ideal. El principio de funcionamiento y el ciclo termodinámico del motor Robinson se pueden consultar en la referencia (Senft, 1993).

Tabla 1 Datos del motor Robinson usado en este estudio

\begin{tabular}{|l|l|l|l|}
\cline { 2 - 4 } \multicolumn{1}{l|}{} & Símbolo & Unidades & Valor \\
\hline Carrera del pistón & $\mathrm{X}_{\mathrm{P}}$ & $\mathrm{m}$ & $5.08 \times 10^{2}$ \\
\hline Carrera del desplazador & $\mathrm{X}_{\mathrm{D}}$ & $\mathrm{m}$ & $5.08 \times 10^{2}$ \\
\hline Diámetro interior de la camisa del pistón & $\mathrm{D}_{\mathrm{P}}$ & $\mathrm{m}$ & $3.56 \times 10^{2}$ \\
\hline Diámetro interior de la camisa del desplazador & $\mathrm{D}_{\mathrm{D}}$ & $\mathrm{m}$ & $5.97 \times 10^{2}$ \\
\hline Volumen muerto en el motor & $\mathrm{V}_{\mathrm{m}}$ & $\mathrm{m}^{3}$ & $7.90 \times 10^{5}$ \\
\hline Temperatura de calentamiento & $\mathrm{T}_{\mathrm{H}}$ & $\mathrm{K}$ & 523 \\
\hline Temperatura de enfriamiento & $\mathrm{T}_{\mathrm{L}}$ & $\mathrm{K}$ & 298 \\
\hline Temperatura en el regenerador $\mathrm{T}_{\mathrm{ml}}$ & $\mathrm{T}_{\mathrm{R}}$ & $\mathrm{K}$ & 400 \\
\hline Presión máxima & $\mathrm{P}_{\operatorname{máx}}$ & $\mathrm{kPa}$ & 115.06 \\
\hline Presión mínima & $\mathrm{p}_{\min }$ & $\mathrm{kPa}$ & 80.5 \\
\hline Velocidad angular & $\omega$ & $\mathrm{rad} / \mathrm{s}$ & 104.7 \\
\hline $\begin{array}{l}\text { Angulo de desfasamiento entre cilindros } \\
\text { para el inicio de los cálculos }\end{array}$ & $\alpha$ & $\circ$ & 90 \\
\hline Constante R del gas a emplear & $\mathrm{R}$ & $\mathrm{kJ} / \mathrm{kg} \mathrm{K}$ & 0.287 \\
\hline Calor específico a presión constante & $\mathrm{C}_{\mathrm{p}}$ & $\mathrm{kJ} / \mathrm{kg} \mathrm{K}$ & 1.019 \\
\hline Calor específico a volumen constante & $\mathrm{C}_{\mathrm{v}}$ & $\mathrm{kJ} / \mathrm{kg} \mathrm{K}$ & 0.728 \\
\hline
\end{tabular}




\section{METODOLOGÍA DE CÁLCULO DE LOS PRINCIPALES PARÁMETROS}

Volumen barrido por el pistón

$$
V_{P}=\frac{\pi}{4} \cdot D_{P}^{2} \cdot X_{P}=5.06 \cdot 10^{-5}\left[\mathrm{~m}^{3}\right]
$$

Volumen barrido por el desplazador

$$
\mathrm{V}_{\mathrm{D}}=\frac{\pi}{4} \cdot \mathrm{D}_{\mathrm{D}}^{2} \cdot \mathrm{X}_{\mathrm{D}}=\mathrm{V}_{\mathrm{D}}=1.42 \cdot 10^{-4}\left[\mathrm{~m}^{3}\right]
$$

A partir de los planos del motor se determina el volumen muerto, resultando ser para nuestro caso de estudio de $V_{m}=7.9 \times 10{ }^{5} \mathrm{~m}^{3}$.

Volumen total del motor

$$
V_{T}=V_{P}+V_{D}+V_{m}=2.72 \cdot 10^{-4}\left[m^{3}\right]
$$

Conociendo este dato se procede a calcular el valor total de la masa para el volumen total en el interior del motor, por medio de la ecuación de estado. Para el caso de estudio se obtiene un valor de $\mathrm{m}=2.6 \times 10^{-4} \mathrm{~kg}$ masa de aire.

La razón entre las temperaturas de calentamiento y enfriamiento, entre los volúmenes barridos por el pistón y el desplazador, y entre volumen muerto y barrido por el desplazador son $\tau=0.57, k=0.356$ y $\chi=0.555$, respectivamente.

La aplicación del modelo Senft-Schmidt inicia con el cálculo de la presión media en base a las razones de temperaturas y volúmenes para lo que se requieren las ecuaciones de los parámetros $\mathrm{Y}$ y X:

$$
\mathrm{Y}=1+\tau+\kappa+\frac{4 \chi \tau}{1+\tau}=2.73
$$

$X=\sqrt{\kappa^{2}-2 \kappa(1-\tau) \cos \alpha+(1-\tau)^{2}}=0.56$

Sustituyendo los valores de $\mathrm{Y}$ y $\mathrm{X}$ en la siguiente ecuación se obtiene la presión media en el motor

$$
\bar{p}=\frac{m R T_{L}}{\left(\frac{V_{D}}{2}\right) \sqrt{Y^{2}-X^{2}}}=115.06 \mathrm{kPa}
$$

Con los valores obtenidos anteriormente de $\mathrm{Y}, \mathrm{X}$ y $\overline{\mathrm{p}}$, se calcula el trabajo indicado del ciclo

$$
W_{\text {ind }}=\frac{V_{T} \bar{p}}{\kappa+1} \frac{\pi(1-\tau) \kappa \operatorname{sen} \alpha}{\left(\sqrt{Y^{2}-X^{2}}+Y\right)}=2.05 \cdot 10^{-3} \mathrm{~kJ}
$$


Hasta aquí se ha obtenido el valor del trabajo indicado por el método Senft-Schmidt. Ahora se calcula el trabajo reversible, para esto se requieren las cantidades de calor suministrado y rechazado para el cambio de volumen por proceso, $V_{1}=2.21 \times 10^{4} \mathrm{~m}^{3}$ del proceso de compresión (el pistón se encuentra en el P.M.S.) y $V_{2}=V_{T}=2.72 \times 10^{4} \mathrm{~m}^{3}$ del proceso de expansión (el pistón se encuentra en el P.M.I.), las cuales son:

Calor suministrado

$$
\mathrm{Q}_{\mathrm{sum}}=\mathrm{m} \cdot \mathrm{R} \cdot \mathrm{T}_{\mathrm{H}} \cdot \ln \left(\frac{\mathrm{V}_{2}}{\mathrm{~V}_{1}}\right)=7.91 \cdot 10^{-3} \mathrm{~kJ}
$$

\section{Calor rechazado}

$$
\mathrm{Q}_{\text {rech }}=\mathrm{m} \cdot \mathrm{R} \cdot \mathrm{T}_{\mathrm{L}} \cdot \ln \left(\frac{\mathrm{V}_{1}}{\mathrm{~V}_{2}}\right)=-4.5 \cdot 10^{-3} \mathrm{~kJ}
$$

La diferencia entre el calor absorbido y el calor rechazado por el ciclo tiene un valor de $3.403 \mathrm{~kJ}$, esta diferencia debería ser la cantidad de trabajo disponible del motor de acuerdo a la expresión $W=Q_{\text {sum }} Q_{\text {rech }}$, sin embargo, no es utilizado en su totalidad por el motor debido a las irreversibilidades. Considerando que el trabajo disponible, $\mathrm{W}$, es el trabajo reversible $\mathrm{W}_{\text {rev }}$, se puede calcular $\mathrm{W}_{\text {perd }}$ como el trabajo disponible no aprovechado. Lo anterior se realiza mediante la diferencia entre el trabajo reversible $W_{\text {rev }}$ y el trabajo irreversible $W_{\text {irrev }}$, considerando a éste último como el trabajo indicado $W_{\text {ind }}$.

$$
\mathrm{W}_{\text {perd }}=\mathrm{W}_{\text {rev }}-\mathrm{W}_{\text {ind }}=1.35 \cdot 10^{-3} \mathrm{~kJ}
$$

A continuación se calcula la eficiencia térmica con el valor del calor total suministrado, obtenido al emplear los valores de la entalpía para el aire a la máxima y mínima temperaturas, y del trabajo indicado calculado para el diseño original del motor:

$$
\eta=\frac{W_{\text {ind }}}{Q_{\text {tot_sum }}}=\frac{2.05 \cdot 10^{-3} \mathrm{~kJ}}{5.8 \cdot 10^{-2} \mathrm{~kJ}} \times 100=3.5 \%
$$

Se considera la temperatura ambiente como el valor de referencia y el trabajo disponible obtenido anteriormente para calcular la entropía generada aplicando el teorema de Gouy-Stodola. La entropía así obtenida servirá como referencia para comparar los valores de entropía calculados posteriormente:

$$
\mathrm{S}_{\text {gen }}=\frac{\mathrm{W}_{\text {perd }}}{\mathrm{T}_{0}}=4.54 \cdot 10^{-6}[\mathrm{~kJ} / \mathrm{K}]
$$

El valor de la entropía generada se debe disminuir para incrementar la eficiencia en el motor. Para la optimización es necesario disminuir los volúmenes, principalmente del desplazador, acompañado de la reducción de los espacios muertos en el motor. Senft (2002) sugiere que la razón entre el volumen del pistón $V_{P}$ y el volumen del desplazador $V_{D}$, debe ser lo más próxima a la unidad para estimar un valor medio en la eficiencia térmica. Por lo anterior, en la segunda iteración de este ejemplo se iguala el valor del espacio del desplazador al del pistón, es decir $V_{P}=V_{D}=5.06 \times 10^{5} \mathrm{~m}^{3}$. Además, se reduce el volumen muerto $V_{m}$ a un valor menor al inicial resultado de $7.6 \times 10^{6} \mathrm{~m}^{3}$.

Si en los cálculos subsiguientes la entropía generada es mayor que su valor de referencia, se deberá disminuir la cantidad de masa hasta que disminuya la entropía respecto a la de referencia. La entropía 
generada es el parámetro que marca el límite inferior al que se puede disminuir la cantidad de masa que debe contener el motor, si se pasa ese límite se tendrán valores negativos de la entropía.

Por otra parte, si el valor de la entropía es menor al de referencia, se hace variar la cantidad de trabajo indicado mediante la disminución del volumen total, para lograr que aumente el valor de la eficiencia. Si la eficiencia térmica disminuye, se deberá disminuir el volumen total. Si la eficiencia térmica aumenta, se deberá verificar que no varíe la entropía generada. Al presentarse lo último se deberá tomar un valor anterior del volumen total hasta obtener un valor máximo de la eficiencia térmica.

Finalmente, al haber conseguido disminuir la entropía e incrementado la eficiencia se deberá variar el ángulo $\alpha$ ubicado entre los ejes del pistón y del desplazador para incrementar el trabajo indicado a un valor máximo. De acuerdo a las recomendaciones proporcionadas por Senft (2002), se hace variar el ángulo $\alpha$ desde $90^{\circ}$ a $95^{\circ}$. Lo anterior es con la finalidad de incrementar la cantidad de trabajo. Una vez obtenido el valor del trabajo con el nuevo ángulo $\alpha$, se verifica su incremento al igual que el de la eficiencia térmica, y se revisa que el valor de la entropía generada haya permanecido constante. En la figura 1 se presenta el diagrama de flujo de la metodología empleada en conjunción con la optimización fundada en la mínima generación de entropía.

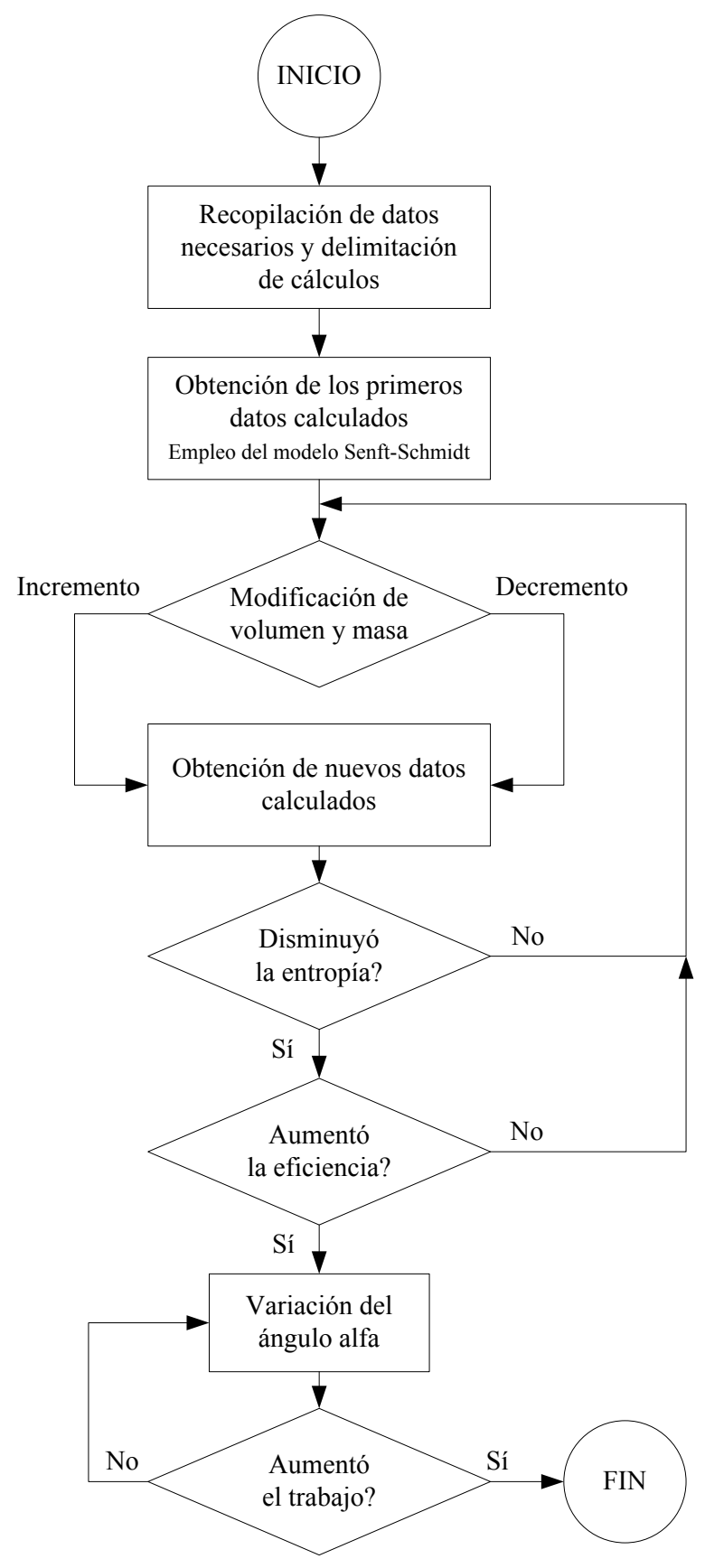

Fig. 1: Diagrama de flujo de la metodología propuesta. 


\section{ANÁLISIS DE RESULTADOS}

En la Tabla 2 se muestran los resultados de cuatro iteraciones realizadas para optimizar la eficiencia térmica del motor Robinson estudiado en este trabajo. Se puede apreciar que la eficiencia del motor, $\eta$, se incrementa cuando se reducen la cantidad de masa, $\mathrm{m}$, y el volumen instantáneo total, $\mathrm{V}_{\mathrm{T}}$. Estos dos parámetros son cruciales para el diseño del motor pues el primero se refiere a la cantidad óptima de fluido de trabajo que hay que suministrar y en el segundo están incluidos los volúmenes o espacios muertos. De igual forma, se puede notar que la entropía generada, $S_{\text {gen }}$, disminuyó al igual que la cantidad de calor a suministrarse, $Q_{\text {sum }}$, lo que condujo a un incremento de la eficiencia, por una parte, y a una disminución de la energía térmica que hay que suministrar al motor.

Hay que hacer notar que para el cálculo 4, quinta columna en la Tabla 2, se muestra los resultados que corresponden a un ángulo $\alpha$ de $95^{\circ}$, esta variación se refleja en el incremento del trabajo indicado, $W_{\text {ind }}$, de $9.91 \times 10^{4} \mathrm{~kJ}$ a $9.96 \times 10^{4} \mathrm{~kJ}$. Esto a su vez condujo a un incremento de la eficiencia térmica de 7.91 a 7.95 $\%$

Resumiendo, se encontró que al emplear el modelo de Senft-Schmidt-Petrescu en conjunto con la optimización fundada en la mínima generación de entropía, la eficiencia térmica, $\eta$, se incrementó de 3.5 a $7.95 \%$. Este incremento de la eficiencia en $4.45 \%$ se logró con una reducción del volumen muerto, $\mathrm{V}_{\mathrm{m}}$, de $9.6 \%$, una disminución de la cantidad de masa del fluido de trabajo, $\mathrm{m}$, en $21 \%$ de su valor original y un decremento de la entropía generada, $S_{\text {gen }}$, de $10 \%$. Estos resultados pueden servir de base en el rediseño del prototipo del motor Robinson del caso de estudio.

Tabla 2: Resultados de las variables de rediseño para cuatro diferentes cálculos.

\begin{tabular}{|c|c|c|c|c|c|}
\hline Variable & Cálculo 1 & Cálculo 2 & Cálculo 3 & Cálculo 4 & Unidades \\
\hline $\mathrm{m}$ & $2.6 \times 10^{4}$ & $1.02 \times 10^{4}$ & $5.48 \times 10^{5}$ & $5.48 \times 10^{5}$ & $\mathrm{~kg}$ \\
\hline $\mathrm{V}_{\mathrm{T}}$ & $2.72 \times 10^{4}$ & $1.09 \times 10^{4}$ & $1.09 \times 10^{4}$ & $1.09 \times 10^{4}$ & $\mathrm{~m}^{3}$ \\
\hline $\mathrm{V}_{\mathrm{P}}$ & $5.06 \times 10^{5}$ & $5.06^{5}$ & $5.06 \times 10^{5}$ & $5.06 \times 10^{5}$ & $\mathrm{~m}^{3}$ \\
\hline $\mathrm{V}_{\mathrm{D}}$ & $1.42 \times 10^{4}$ & $5.06 \times 10^{5}$ & $5.06 \times 10^{5}$ & $5.06 \times 10^{5}$ & $\mathrm{~m}^{3}$ \\
\hline $\mathrm{V}_{\mathrm{m}}$ & $7.9 \times 10^{5}$ & $7.6 \times 10^{6}$ & $7.6 \times 10^{6}$ & $7.6 \times 10^{6}$ & $\mathrm{~m}^{3}$ \\
\hline$\overline{\mathrm{p}}$ & 115.06 & 134.88 & 72.15 & 72.58 & $\mathrm{kPa}$ \\
\hline $\mathrm{W}_{\text {ind }}$ & $2.05 \times 10^{3}$ & $1.85 \times 10^{3}$ & $9.91 \times 10^{4}$ & $9.96 \times 10^{4}$ & $\mathrm{~kJ}$ \\
\hline $\mathrm{W}_{\text {rev }}$ & $3.4 \times 10^{3}$ & $4.14 \times 10^{3}$ & $2.21 \times 10^{3}$ & $2.21 \times 10^{3}$ & $\mathrm{~kJ}$ \\
\hline $\mathrm{W}_{\text {perd }}$ & $1.35 \times 10^{3}$ & $2.29 \times 10^{3}$ & $1.22 \times 10^{3}$ & $1.22 \times 10^{3}$ & $\mathrm{~kJ}$ \\
\hline $\mathrm{Q}_{\text {sum }}$ & $7.91 \times 10^{3}$ & $9.62 \times 10^{3}$ & $5.14 \times 10^{3}$ & $5.15 \times 10^{3}$ & $\mathrm{~kJ}$ \\
\hline $\mathrm{Q}_{\text {rech }}$ & $4.5 \times 10^{3}$ & $5.48 \times 10^{3}$ & $2.93 \times 10^{3}$ & $2.93 \times 10^{3}$ & $\mathrm{~kJ}$ \\
\hline $\mathrm{S}_{\text {gen }}$ & $4.54 \times 10^{6}$ & $7.67 \times 10^{6}$ & $4.1 \times 10^{6}$ & $4.1 \times 10^{6}$ & $\mathrm{~kJ} / \mathrm{K}$ \\
\hline$\eta$ & 3.5 & 7.91 & 7.91 & 7.95 & $\%$ \\
\hline
\end{tabular}

\section{CONCLUSIONES}

En este trabajo se presentó un modelo matemático basado en los modelos de Senf Schmidt y de Petrescu, los cuales están ya establecidos para analizar los aspectos de pérdidas totales en el desarrollo del trabajo útil y la cantidad máxima de trabajo útil, respectivamente, en motores de ciclo Stirling. Del análisis de resultados de este trabajo se pudo llegar a las siguientes conclusiones:

1) Con el modelo Senf Schmidt Petrescu, como se le llamó al modelo propuesto, es posible optimizar la eficiencia térmica de los motores de ciclo Stirling tipo $\gamma$ y motores Robinson, al reducir la generación de entropía fijando los parámetros esenciales que modifican el diseño del motor, siendo éstos la cantidad de masa del fluido de trabajo y el volumen total incluyendo el espacio muerto.

2) Las expectativas para aplicar el modelo de Senf Schmidt Petrescu en otro tipo de motores de ciclo Stirling, como el tipo $\beta$, quedan abiertas para usar las ecuaciones correspondientes a esas variantes.

\section{AGRADECIMIENTOS}

Los autores agradecen al Instituto Politécnico Nacional de México por el financiamiento otorgado. Un especial agradecimiento se le extiende al Ing. José de la Herrán por haber proporcionado los esquemas básicos para construir el prototipo del motor tipo gama, variante Robinson, que se presenta en este trabajo. 


\section{NOTACIÓN}

a

b

f

$m$

P.M.I.

P.M.S.

$\overline{\mathrm{p}}$

$\dot{\mathrm{Q}}$

$\mathrm{R}$

$\mathrm{S}$

$\mathrm{T}$

$t$

V

$\mathrm{V}$

W

W

$\mathrm{X}$

$\mathrm{X}$

Y

\section{Letras griegas}

$\alpha$

$\varepsilon$

$\eta$

$\kappa$

$\tau$

$\chi$

\section{Subíndice}

D

est

$\mathrm{f}$

gen

$\mathrm{H}$

ind

i

irrev

$\mathrm{L}$

m

mod

$P$

perd

rech

rev

sum

$\mathrm{T}$

0

1

2 coeficiente $(=\sqrt{3 \gamma})$

coeficiente $\in[0,2]$

coeficiente de fricción $\in[0,1]$

masa

[kg]

punto muerto inferior

punto muerto superior

presión media

[Pa]

flujo de calor total

[kW]

constante de gas

$[\mathrm{kJ} / \mathrm{kg} \mathrm{K}]$

entropía

temperatura

$[\mathrm{kJ} / \mathrm{K}]$

[K]

[s]

volumen

volumen específico

trabajo

$\left[\mathrm{m}^{3}\right]$

$\left[\mathrm{m}^{3} / \mathrm{kg}\right]$

[kJ]

[kW]

relación entre razón de volúmenes y temperatura respecto al ángulo entre cilindros.

desplazamiento lineal del pistón en cualquier instante

relación entre razón de temperaturas y de volúmenes

incluyendo el volumen muerto

$[\mathrm{m}]$

ángulo de desfasamiento particular entre pistón y desplazador

relación de compresión

eficiencia térmica

relación entre volúmenes barridos

relación entre temperaturas máxima y mínima

razón del volumen muerto

desplazador

Estrangulamiento

fricción

generado

fuente

indicado

instantaneo

irreversible

sumidero

volumen muerto

modelo

pistón

perdido

rechazado

reversible

suministrado

total

valor de referencia

volumen cuando el pistón se encuentra en el P.M.S.

volumen cuando el pistón se encuentra en el P.M.I.

\section{REFERENCIAS}

Bejan, A., Advanced engineering thermodynamics, $3^{\text {a }}$ edición. Wiley, New Jersey (2006).

Campos, M.C., J.V.C. Vargas y J.C. Ordonez, Thermodynamic optimization of a Stirling engine, Energy: 44(1), 902-910 (2012). 
Chen, N.C.J. y F.P. Griffin, A review of Stirling engine mathematical models (1983), http://www.ornl.gov/info/reports/1983/3445602862052.pdf. Acceso: 28 de Febrero (2013).

Cheng, C. H. y H. S. Yang, Optimization of geometrical parameters for Stirling engines based on theoretical analysis, Applied Energy: 92(3) 395-405 (2012).

De Boer, P.C.T., Maximum attainable performance of Stirling engines and refrigerators, J. Heat Transfer: 125(5) 911-915 (2003).

Ferreira, A. C. M., M. L. Nunes, L. A. S. B. Martins y F. C. F. Teixeira, A review of Stirling engine technologies applied to micro cogeneration systems, Actas del $25^{\text {th }}$ International Conference on Efficiency, Cost, Optimization, Simulation and Environmental Impact of Energy Systems, 1-11, Perugia, Italia, 26 al 29 de Junio (2012).

Gheith, R., F. Aloui, M. Tazerout y S. Ben Nasrallah, Experimental investigations of a gamma Stirling engine, Int. J. Energy Research: 36(12) 1175-1182 (2012).

Karabulut, H., F. Aksoy y E. Öztürk, Thermodynamic analysis of a type Stirling engine with a displacer driving mechanism by means of a lever, Renewable Energy: 34(1) 202-208 (2009).

Khan, M. U., Efficiency and emissions study of a residential micro cogeneration system based on a modified Stirling engine and fuelled by a wood derived fast pyrolysis liquid ethanol blend, Tesis de Magister, Dep. of Mech. and Ind. Eng., University of Toronto, Toronto, Canada (2012).

Kongtragool, B. y S. Wongwises, A four power piston low temperature differential Stirling engine using simulated solar energy as a heat source, Solar Energy: 82(6) 493-500 (2008).

Miranda, S., I. Carvajal y D. Omaña, Theoretical study of the performance of Stirling engine regenerators, Actas del $15^{\text {th }}$ International Stirling Engine Conference, 1-8, Sesión en línea, 10 al 22 de Septiembre (2012).

Miranda, S., I. Carvajal y J. Vazquez, Methodology for the redesign of Robinson type Stirling engines, Actas del $15^{\text {th }}$ International Stirling Engine Conference, 1-8, Sesión en línea, 10 al 22 de Septiembre (2012).

Organ, A. J., Thermodynamics and gas dynamic of Stirling cycle machine, $1^{\text {a }}$ edición. Cambridge University Press, Cambridge (1992).

Petrescu, S., M. Costea, C. Harman y T. Florea, Application of the direct method to irreversible Stirling cycles with finite speed, Int. J. Energy Research: 26(7) 589-609 (2002).

Petrescu, S., C. Petre, M. Costea, O. Malancioiu, N. Boriaru, A. Dobrovicescu, M. Feidt y C. Harman A methodology of computation, design and optimization of solar Stirling power plant using hydrogen/oxygen fuel cells, Energy: 35(2) 729-739 (2010).

Santos, D., J. Alonso, J. Eloy y S. Arnalte, Solar dish-Stirling system optimisation with a doubly fed induction generator, IET Renewable Power Generation: 6(4) 276-288 (2012).

Senft, J. R., An introduction to Stirling engines, $1^{\text {a }}$ edición. Moriya Press, River Falls, Wisconsin (1993).

Senft, J. R., Optimum Stirling engine geometry, Int. J. Energy Research: 26(12), 1087-1101 (2002).

Senft, J. R., Mechanical Efficiency of Heat Engines, $1^{\text {a }}$ edición. Cambridge University Press, Cambridge (2011).

Siva Reddy, V., S. C. Kaushik y S. K. Tyagi, Year round performance and economic evaluation of solar power plant for Indian tropical condition, J. Renewable Sustainable Energy: 4(4) 1-12 (2012). 